\title{
Tool for rapid annotation of microbial SNPs (TRAMS): a simple program for rapid annotation of genomic variation in prokaryotes ${ }^{1}$
}

\section{Richard A. Reumerman, Nicholas P. Tucker, Paul R. Herron, Paul A. Hoskisson, Vartul Sangal}

University of Strathclyde, Glasgow; E-mail: Richard.Reumerman@strath.ac.uk

\section{TRAMS provides rapid and accurate SNP annotation to NGS users with varying computational experience}

Next-generation sequencing (NGS) has been widely applied to a variety of microorganisms for evolutionary and epidemiological investigations. Genome wide variation (single nucleotide polymorphism, SNP; multiple nucleotide polymorphism, MNP) from NGS data analyses need functional annotation for their impact on coding sequences. We have developed a program, TRAMS, to annotate large numbers of SNPs between several strains

TRAMS is especially designed for growing number of NGS users with limited computational experience who can use an executable file on WINDOWS computers without needing any installation or command lines to run. TRAMS is available as a Python $^{2}$ script for MacOS and Linux users and also works within Galaxy ${ }^{3}$, a highly used tool for analysing NGS data. TRAMS accurately annotated >70,00o SNPs between four Corynebacterium diphtheriae strains and will be very useful in analysing microbial genomic diversity.

\section{SNPs are extracted from the genome alignment and functionally annotated using a reference genome}

Four published $C$. diphtheriae genomes ${ }^{47}$ were aligned (Fig 1a) and SNPs were extracted using Mauve ${ }^{8}$. An input file (Fig $1 \mathrm{~b}$ )containing the SNP positions, reference alleles and SNP alleles in a tab delimited format was generated using a script, mauveztrams.py, that is also included in the TRAMS package. SNPs were annotated against the reference genome in the GenBank format using TRAMS. WINDOWS users can use the executable version of TRAMS and enter the input file names when prompted (Fig 1C). MacOS and Linux users can download the scripts and run it by a command line (Fig. 1d).
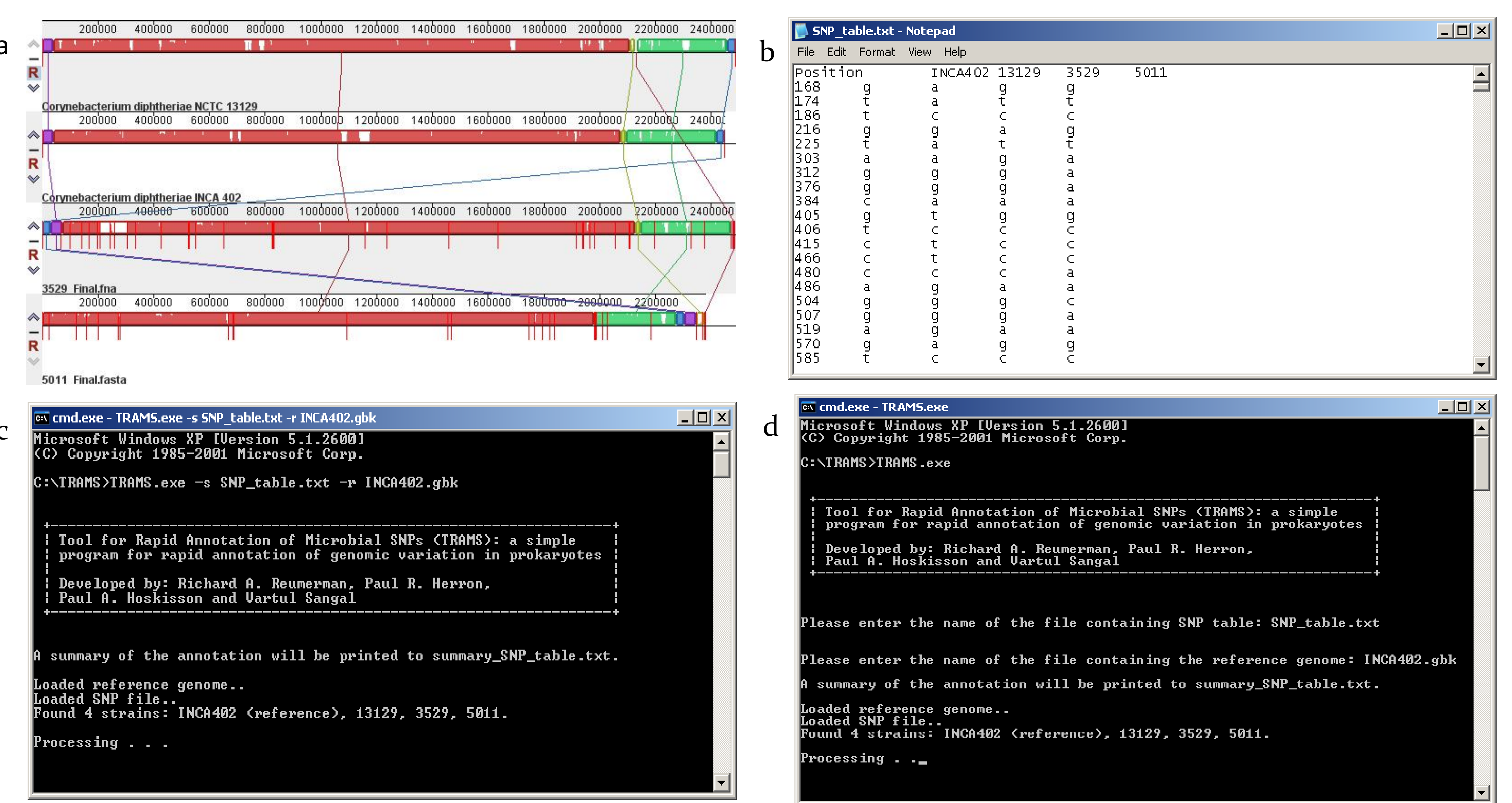

Fig 1: genome alignment (a), extracted SNPs (b) and annotation usint TRAMS (c,d)

\section{TRAMS output: Annotation, summary and list of} SNPs in overlapping features

Three output files are produced (Fig 2a-c). The annotation file contain the SNP types for each strain, the summary file contain an overview of SNP properties and the overlap file lists the SNPs that are present in overlapping features.

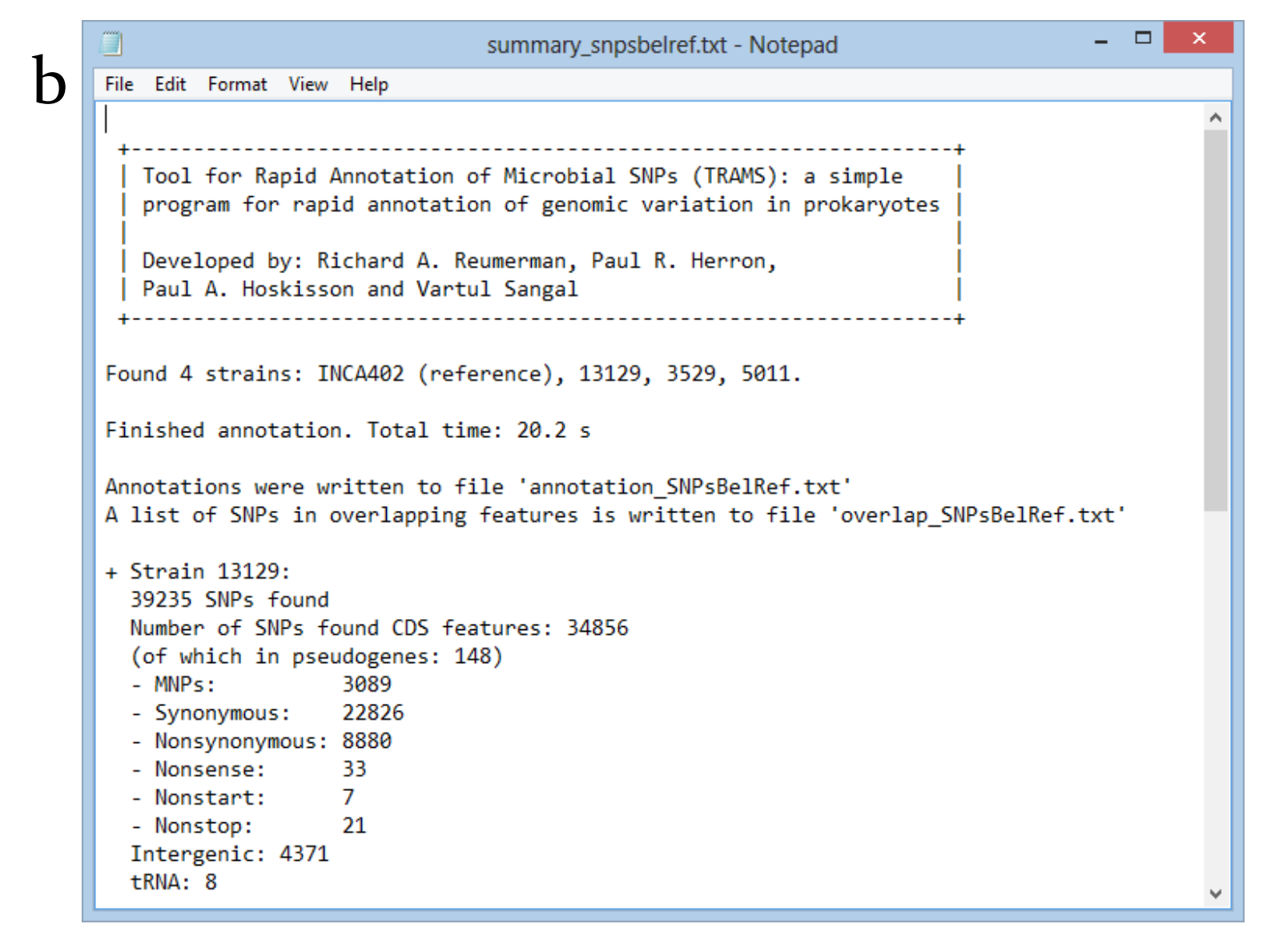

\section{TRAMS can be used in Galaxy workflows}

Galaxy is a bioinformatics platform that enables use of a growing number of bioinformatic tools through a single graphic user interface. Workflows can be set up that allow multiple tools to be used consecutively, enabling automated and reproducible data analysis. TRAMS was adapted to work in this environment and is available through the Galaxy toolshed for installation to local galaxy instances.

The figure below shows an example of a workflow that maps Illumina sequence data to a reference genome, extracts SNPs and annotates them using TRAMS.

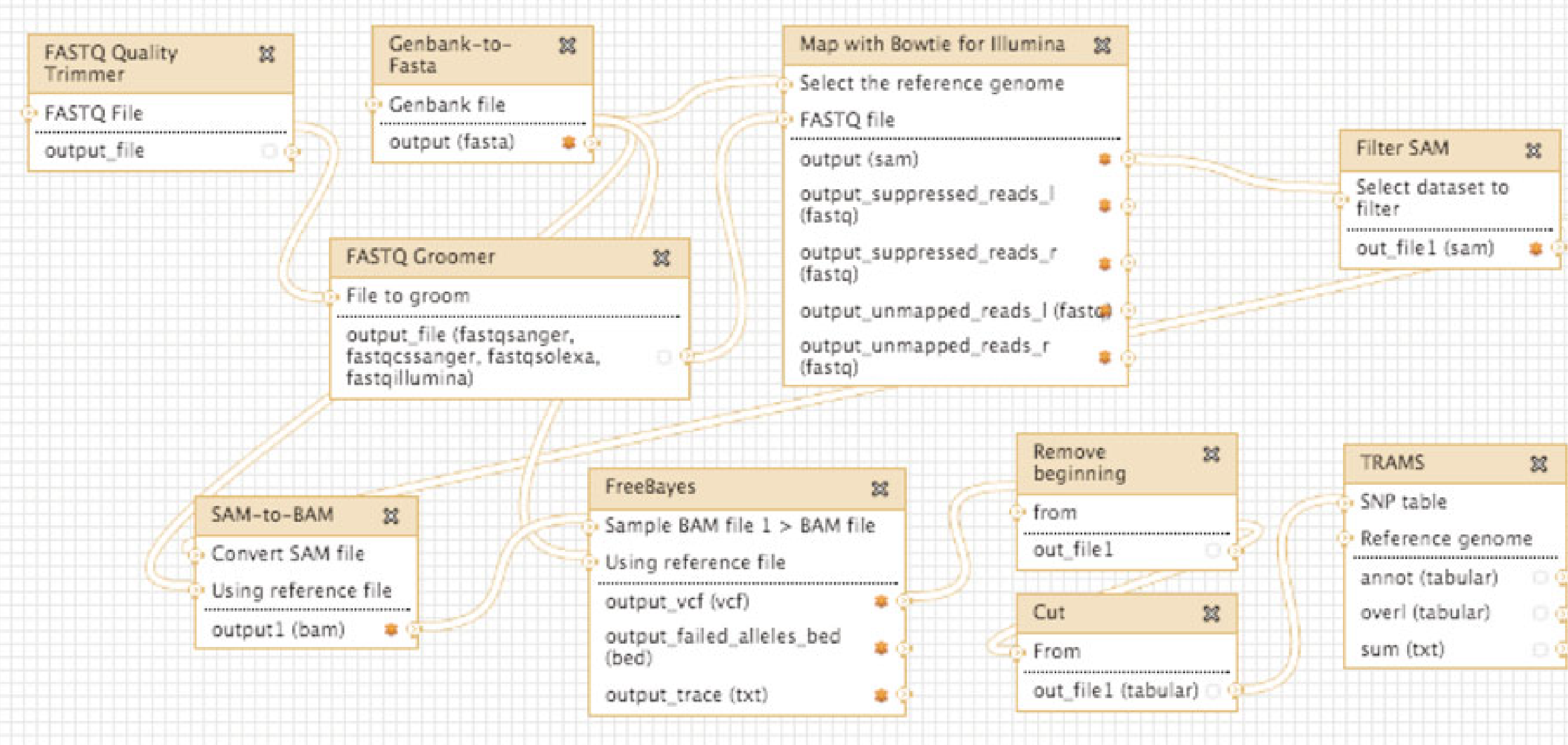

Fig 3: Galaxy workflow mapping sequencing reads to a reference genome, extracting SNPs and annotating them using TRAMS

\section{Conclusions}

We have developed a program (TRAMS) that:

- Can functionally annotate large numbers of SNPs

- Can be used using at least three platforms (Windows,

Linux, MacOS)

- Is easy to use, as it requires no installation (when used on windows) and does not require complex command line arguments

- Annotates SNPs as intergenic, synonymous or nonsynonymous but subdivides nonsynonymous SNPs into nonsense, nonstart or nonstop SNPs

- Is available for Galaxy from the main Galaxy toolshed

\section{Availability}

TRAMS can be downloaded as a Python script or Windows executable from http://sourceforge.net/projects/strathtrams/. Instructions are included with the program.

The Python script requires Python (ver. 2.7) and the BioPython package $^{2}$ (ver. 1.6o) to be installed. Python can be downloaded from www.python.org, Biopython is available from www.biopython.org.

\section{References}

1: Reumerman, R.A. et al., 2013, Antonie van Leeuwenhoek 104(3) pp. 431-434

2: Cock, P.J. et al., 2009, Bioinformatics 25(11) pp. 1422-1423

3: Blankenberg, D. et al., 2010, Curr. Protoc. Mol. Biol. Ch.19,

19.10.11-21

4: Sangal, V. et al., 2012a, J. Bacteriol. 194(12) p. 3269

5: Sangal, V. et al., 2012b, J. Bacteriol. 194(17) p. 4738

6: Trost, E. et al., 2012, J. Bacteriol. 194(12) pp.3199-3215

7: Cerdeno-Tarraga, A.M. et. al., 2003, Nucleic Acids Res. 31(22)

pp. 6516-6523

8: Darling, A.C. et al., 2004, Genome Res. 14(7) pp. 1394-1403 